\title{
Association between glutathione S-transferase M1/T1 gene polymorphisms and susceptibility to endometriosis: A systematic review and meta-analysis
}

\author{
XIAOYAN XIN ${ }^{1 *}$, ZHISHAN JIN $^{1 *}$, HUAJIAN GU ${ }^{2}$, YUANYUE LI $^{1}$, \\ TINGTING WU ${ }^{1}$, TENG HUA ${ }^{1}$ and HONGBO WANG ${ }^{1}$
}

${ }^{1}$ Department of Gynecology and Obstetrics, Union Hospital, Tongji Medical College, Huazhong University of Science and Technology, Wuhan, Hubei 430022; ${ }^{2}$ Department of General Surgery, Affiliated Hospital of Guiyang Medical College, Guiyang, Guizhou 550001, P.R. China

Received December 29, 2014; Accepted February 11, 2016

DOI: 10.3892/etm.2016.3110

\begin{abstract}
Endometriosis is a polygenic/multifactorial disease caused by interactions between multiple genes and the environment. Findings from studies evaluating the association between the glutathione S-transferase (GST) M1/T1 null genotype and susceptibility to endometriosis are inconsistent. This meta-analysis updated and reevaluated the possible associations between GSTM1, GSTT1 and combined GSTM1/GSTT1 (null genotype versus wild-type) gene polymorphisms and susceptibility to endometriosis. The PubMed, Embase and Chinese BioMedical Literature databases and Google Scholar were searched for case-control genetic association studies on GSTM1/GSTT1 (null genotype versus wild-type) gene polymorphisms and endometriosis in comparison with non-endometriosis or healthy controls. Fixed-effect and random-effect meta-analytical techniques were conducted for the outcome measure and subgroup analyses. The meta-analysis demonstrated significant associations between the GSTM1 [odds ratio $(\mathrm{OR})=1.56$; $95 \%$ confidence interval (CI): 1.25-1.95; $\mathrm{P}<0.0001)$, GSTT1 (OR=1.31; 95\% CI: 1.02-1.68; $\mathrm{P}=0.037)$ and GSTM1/GSTT1 (OR=1.68; 95\% CI: 1.29-2.17; $\mathrm{P}<0.0001)$ null genotypes and increased risk for endometriosis. The results suggest that the GSTM1, GSTT1, and combined GSTM1/GSTT1 null genotypes increase susceptibility to endometriosis. Additional well-designed studies and precise analyses are warranted to confirm these findings.
\end{abstract}

Correspondence to: Professor Hongbo Wang, Department of Gynecology and Obstetrics, Union Hospital, Tongji Medical College, Huazhong University of Science and Technology, 1277 Jiefang Avenue, Wuhan, Hubei 430022, P.R. China

E-mail: hb_wang1969@sina.com

*Contributed equally

Key words: gene polymorphisms, glutathione S-transferase M1, glutathione S-transferase T1, endometriosis, meta-analysis

\section{Introduction}

Endometriosis manifests as ectopic endometrial cells outside the uterus. It is an intractable disease that causes infertility, dysmenorrhea and pelvic pain. Endometriosis occurs in $10 \%$ of women of childbearing age. Notably, the incidence of endometriosis has been rising in recent years (1). The pathogenesis of endometriosis remains to be elucidated.

Published reports indicate that endometriosis is a polygenic/multifactorial disease caused by interactions between multiple genes and the environment $(2,3)$. In particular, a correlation has been identified between endometriosis and exposure to environmental toxins such as dioxin (4); dioxin and dioxin-like compounds have been implicated in the development of endometriosis $(5,6)$.

The phase II conjugation enzymes usually function to inactivate environmental toxins. Among these, glutathione S-transferase (GST) may be critical for the detoxification of dioxins. Human GSTs are classified into two distinct categories: Soluble or cytosolic and membrane-bound microsomal. The soluble or cytosolic GSTs are subdivided into seven families named $\alpha, \mu, \omega, \pi, \sigma, \theta$ and $\zeta$ (7). Genes in several of these families are polymorphic, including: GSTA2 in the $\alpha$ family, GSTM1 and GSTM3 in the $\mu$ family, GSTP1 in the $\pi$ family, GSTO, GSTT1, and GSTT2 in the $\theta$ family, and GSTZ1 in the $\zeta$ family. Heritable allelic differences in GSTM1, GSTM3, GSTTI and GSTPI may have marked relevance for individual susceptibility to disease.

GSTM1 and GSTT1 are two candidate genes that may play an important role in the development of endometriosis. GSTM1 and GSTT1 are located on chromosomes 1p13.3 and 22q11.23, respectively. They are critical in the detoxification of the products of oxidative stress produced during the repair of the ovarian epithelium. GSTM1 and GSTT1 null alleles have reduced enzyme activity, a state that may contribute to inefficient detoxification of intermediates produced during stress. This may increase damage to various host genes and contribute to the pathogenesis of endometriosis $(8,9)$.

A meta-analysis summarizing the literature up to the year 2005 suggested that the GSTT1 null genotype, but not the 
GSTM1 null genotype, was associated with an increased risk for endometriosis (7). In the years since 2005, additional reports investigating this topic have been published. The objective of the present study was to update the existing meta-analysis and reevaluate the possible associations between GSTM1, GSTT1 and combined GSTM1/GSTT1 (null genotype vs. wild-type) gene polymorphisms and susceptibility to endometriosis.

\section{Materials and methods}

Searches. For this systematic review and meta-analysis, PubMed (from January 1996 to January 2014), Embase (from January 1996 to January 2014), Chinese BioMedical Literature database (from January 1996 to January 2014) and Google Scholar (from January 1996 to January 2014) were searched. The following keywords were used: 'endometriosis', 'polymorphisms', 'glutathione S-transferases', 'GSTM1' and 'GSTT1' or their combinations.

Reference lists from articles identified by the electronic search were searched by hand. This process was performed iteratively until no additional articles could be identified.

Inclusion and exclusion criteria. Articles published in English or Chinese were included if they reported quantitative outcomes from case-control genetic association studies on GSTM1, GSTT1 or combined GSTM1/GSTT1 (null genotype vs. wild-type) gene polymorphisms and endometriosis versus non-endometriosis or healthy controls.

Studies were excluded if they were case reports, case-only studies, letters, reviews or meta-analyses; included subjects who were related; included cases of adenomyosis, which has unknown etiology (10); reported insufficient data; or were duplicate studies.

Selection of studies. Two reviewers (XYX and ZSJ) independently examined titles and abstracts to select eligible studies. Records were removed that were ongoing or unpublished studies, or were published as abstracts or conference proceedings. Where data sets were overlapping or duplicated, only the most recent information was included. The full text of potentially relevant studies was retrieved. Two reviewers (XYX and HJG) independently examined the full text records to determine which studies met the inclusion criteria. Disagreement about the selection of studies was resolved by discussion and consensus.

Data extraction and management. Two reviewers (XYX and ZSJ) independently extracted data from eligible studies including the first author's last name, publication year, study location, ethnicity, matching variability, diagnostic criteria, stages of disease, source of controls, numbers of cases and controls, and numbers and/or percentages of null genotypes. Disagreement about data extraction was resolved by discussion and consensus.

Assessment of quality of evidence in included studies. Two reviewers (YYL and HJG) independently assessed quality of evidence in the included studies using the 9-star Newcastle-Ottawa Scale, which considers selection, comparability and outcome evaluation criteria.
Assessment of heterogeneity. Heterogeneity was assessed using the $\chi^{2}$ test and $\mathrm{I}^{2}$ test. The $\mathrm{I}^{2}$ statistic was interpreted as follows: $\mathrm{I}^{2}=0-40 \%$, heterogeneity may not be important; $\mathrm{I}^{2}=30-60 \%$, heterogeneity may be moderate; $\mathrm{I}^{2}=50-90 \%$, heterogeneity may be substantial; and $\mathrm{I}^{2}=75-100 \%$, considerable heterogeneity (11). If heterogeneity was present, meta-regression was used to find the source.

Assessment of reporting biases. A funnel plot of effect estimates against their standard errors (SEs) was created to assess possible reporting bias between studies. Funnel plot asymmetry was assessed using Egger's linear regression test and Begg's rank correlation test; $\mathrm{P}<0.05$ suggested publication bias.

GSTM1/GSTT1 and risk for endometriosis. Two reviewers (XYX and HJG) independently combined data from trials using a fixed-effect model (DerSimonian and Laird method) when there was no significant heterogeneity in populations $\left(\mathrm{I}^{2}<50 \%\right)$ and a random-effect model (Mantel-Haenszel method) when there was considerable heterogeneity. Variables were synthesized using odds ratios (ORs). A P-value of 0.05 was used as the cut-off value to determine statistical significance, and data are presented as the estimated OR with 95\% confidence intervals (CIs). All statistical analyses were performed using STATA software, version 12.0 (StataCorp, College Station, TX, USA). Inconsistencies in data analysis were resolved through consensus and discussion with a third reviewer $(\mathrm{ZSJ})$.

Sensitivity and subgroup analyses. Sensitivity analyses were performed to explore the impact of excluding outlying results. Subgroup analyses were performed by stratifying patients according to ethnicity (Caucasian, Asian or mixed), characteristics of controls (hospital patients or healthy individuals), and quality of evidence (high-quality or low-quality).

\section{Results}

Screening and selection. The searches identified 120 articles. Titles and abstracts were screened, and 36 studies were identified as potentially eligible for inclusion. The full text articles for these studies were retrieved. Following analysis of the full text articles, four studies were excluded and 32 studies were found to be eligible for inclusion according to the criteria used for considering studies in this review (Fig. 1).

Included studies. The characteristics of the included studies are shown in Table I. There were 32 case-control genetic association studies involving 3,990 cases of endometriosis and 4,625 controls. One publication addressed two groups of subjects with different ethnicities and was considered as two case-control genetic association studies (12); thus, the total number of studies was considered to be 33. Studies included data relevant to the GSTM1 genotype, GSTT1 genotype or the combined GSTM1/GSTT1 genotype. Of the 32 eligible studies, 20 were conducted in Asia (12-31), eight in Europe (32-39), two in North America $(40,41)$, and two in South America $(42,43)$. The evidence reported in 23 studies was identified as high-quality, and that in 10 studies was identified as low-quality. 


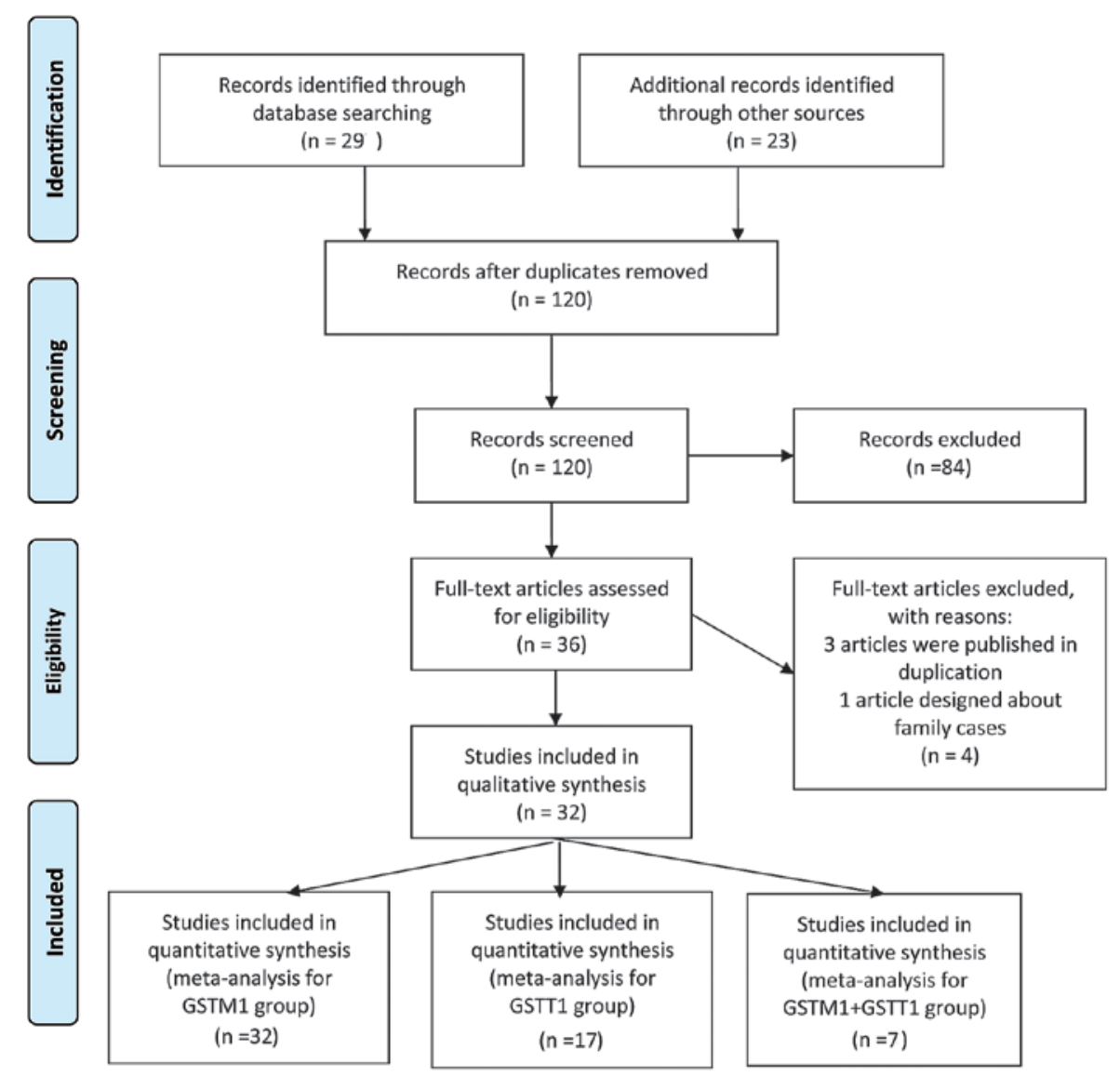

Figure 1. PRISMA 2009 flow diagram showing the article screening and selection process. Using the search strategy, 120 articles were identified by the initial search, and 36 required further assessment. Finally, 32 articles were included in this review, one of which was considered as two studies. GSTM1, glutathione S-transferase $\mu 1$; GSTT1, glutathione S-transferase $\theta 1$.

Excluded studies. Of the 36 studies that were relevant to the GSTM1/GSTT1 genotype and endometriosis, four were excluded. Of these, three were duplicates $(13,14,32)$, and one included subjects who were related (44).

\section{GSTM1/GSTT1 and risk for endometriosis}

GSTMIgenotype. Data reporting on the GSTM1 gene polymorphism are described in 33 case-control studies $(3,990$ cases of endometriosis and 4,625 controls). The meta-analysis demonstrated that there was a significant association between the GSTM1 null genotype and an increased risk for endometriosis (OR=1.56; 95\% CI: 1.25-1.95; $\mathrm{P}<0.0001$; Fig. 2A).

Subgroup analyses stratified by ethnicity (Caucasian: $\mathrm{OR}=1.599$; 95\% CI: 1.205-2.122; $\mathrm{P}=0.001$; Asian: $\mathrm{OR}=1.772$; 95\% CI: 1.242-2.528, $\mathrm{P}=0.002$ ), source of controls (hospital patients: $\mathrm{OR}=1.561 ; 95 \% \mathrm{CI}: 1.151-2.117 ; \mathrm{P}=0.004$; healthy individuals: $\mathrm{OR}=1.569 ; 95 \% \mathrm{CI}: 1.131-2.176 ; \mathrm{P}=0.007)$, and quality of evidence (high-quality: $\mathrm{OR}=1.563$; $95 \% \mathrm{CI}$ : $1.253-1.949$; $\mathrm{P}<0.0001)$ confirmed this finding.

Subgroup analysis stratified for mixed ethnicity (two case control studies involving 111 cases of endometriosis and 78 controls) demonstrated a significant association between the GSTM1 null genotype and a decreased risk for endometriosis (OR=0.404; 95\% CI: 0.219-0.745; $\mathrm{P}=0.004$; Table II). Compared with individual Caucasian and Asian populations, the difference was statistically significant $(\mathrm{P}<0.001$; data shown in Table III).
GSTT1 genotype. Data reporting on the GSTT1 gene polymorphism are described in 18 case-control studies (2,371 cases of endometriosis and 2,490 controls). The meta-analysis demonstrated a significant association between the GSTT1 null genotype and an increased risk for endometriosis $(\mathrm{OR}=1.31$; 95\% CI: 1.02-1.68; P=0.037; Fig. 2B).

Subgroup analysis stratified by ethnicity demonstrated a significant association between the GSTT1 null genotype and an increased risk for endometriosis among Asians (OR=1.573; 95\% CI: 1.186-2.085; $\mathrm{P}=0.002)$, but not among Caucasians (OR=1.124; 95\% CI: 0.745-1.697; $\mathrm{P}=0.577$ ).

Subgroup analyses stratified by the source of controls found no significant association between the GSTT1 null genotype and an increased risk for endometriosis among hospital-based studies $(\mathrm{OR}=1.284 ; 95 \% \mathrm{CI}$ : 0.963-1.712; $\mathrm{P}=0.089)$ or among healthy individuals $(\mathrm{OR}=1.315 ; 95 \% \mathrm{CI}$ : 0.767-2.254; $\mathrm{P}=0.320)$.

Subgroup analyses stratified by quality of evidence demonstrated a significant association between the GSTT1 null genotype and an increased risk for endometriosis among studies considered high-quality evidence ( $\mathrm{OR}=1.376$; 95\% CI: 1.020-1.858; $\mathrm{P}=0.037)$, but not among studies considered low-quality evidence (OR=1.121, 95\% CI: 0.646-1.944; $\mathrm{P}=0.684$; Table II).

Combined GSTM1/GSTT1 genotype. Data reporting on the combined GSTM1/GSTT1 gene polymorphism are described in eight case-control studies (1,083 cases of endometriosis and 1,222 controls). The meta-analysis demonstrated a significant 


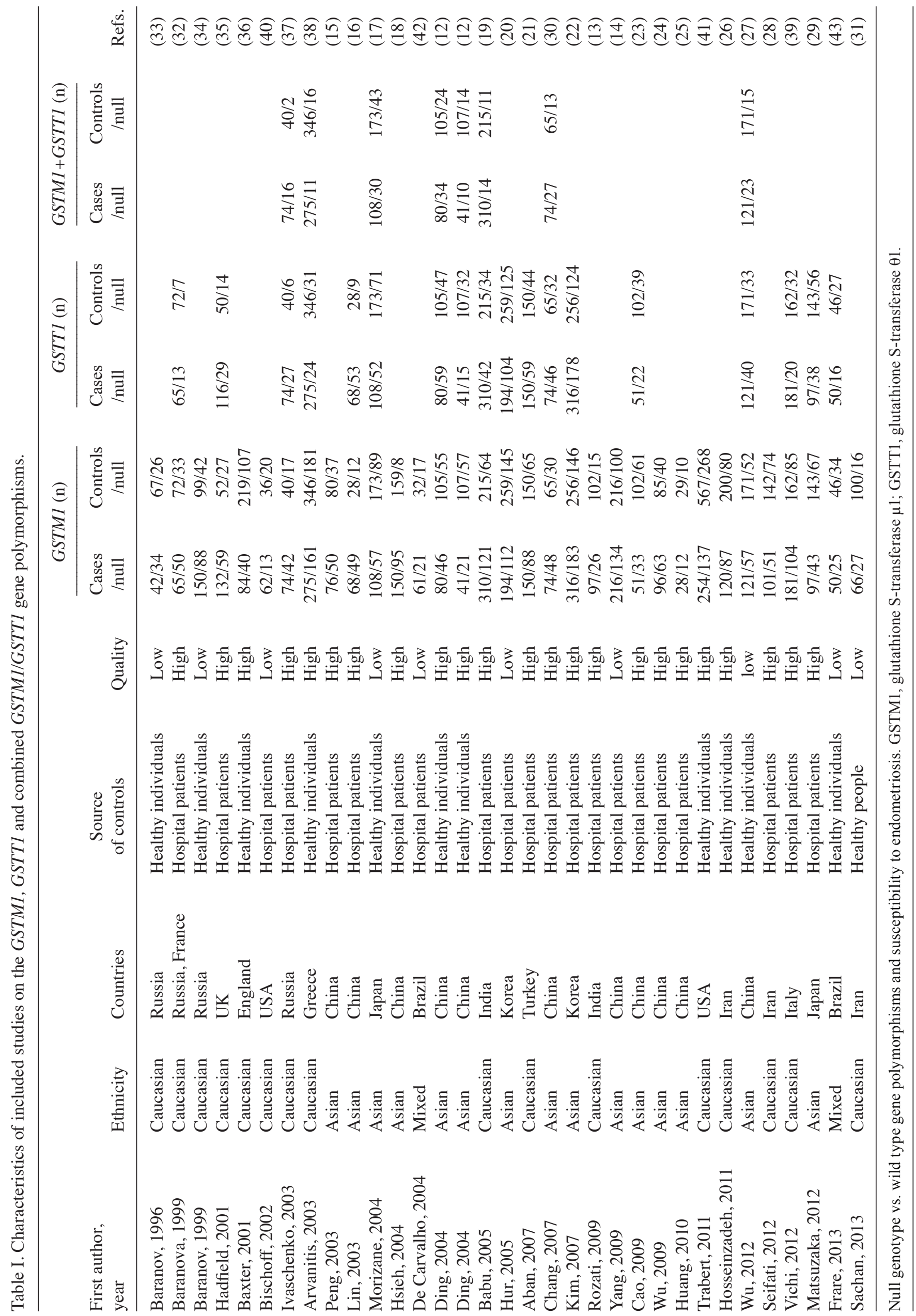


Table II. Meta-analysis of the association between GSTM1, GSTT1 and combined GSTM1/GSTT1 (null genotype vs. wild-type) gene polymorphisms and susceptibility to endometriosis.

\begin{tabular}{|c|c|c|c|c|}
\hline Group & No. of studies & No. of subjects (cases/controls) & OR $[95 \% \mathrm{CI}]$ & P-value \\
\hline \multicolumn{5}{|l|}{ Total studies } \\
\hline GSTM1 genotype & 33 & $3,990 / 4,625$ & $1.563[1.253-1.949]$ & $<0.001$ \\
\hline GSTT1 genotype & 18 & $2,371 / 2,490$ & $1.345[1.044-1.733]$ & 0.022 \\
\hline GSTM1+GSTT1 genotype & 8 & $1,083 / 1,222$ & $1.672[1.291-2.166]$ & 0.005 \\
\hline \multicolumn{5}{|l|}{ Caucasian } \\
\hline GSTM1 genotype & 16 & $2,163 / 2,569$ & $1.599[1.205-2.122]$ & 0.001 \\
\hline GSTT1 genotype & 7 & $1,171 / 1,035$ & $1.124[0.745-1.697]$ & 0.577 \\
\hline GSTM1+GSTT1 genotype & 3 & $659 / 601$ & $1.185[0.717-1.961]$ & 0.508 \\
\hline \multicolumn{5}{|l|}{ Asian } \\
\hline GSTM1 genotype & 15 & $1,716 / 1,978$ & $1.772[1.242-2.528]$ & 0.002 \\
\hline GSTT1 genotype & 10 & $1,150 / 1,409$ & $1.573[1.186-2.085]$ & 0.002 \\
\hline GSTM1+GSTT1 genotype & 5 & $424 / 621$ & $1.898[1.404-2.565]$ & $<0.001$ \\
\hline \multicolumn{5}{|l|}{ Mixed } \\
\hline GSTM1 genotype & 2 & $111 / 78$ & $0.404[0.219-0.745]$ & 0.004 \\
\hline GSTT1 genotype & 1 & $50 / 46$ & & \\
\hline GSTM1+GSTT1 genotype & 0 & & & \\
\hline \multicolumn{5}{|l|}{ Controls from hospital patients } \\
\hline GSTM1 genotype & 21 & $2,599 / 2,425$ & $1.561[1.151-2.117]$ & 0.004 \\
\hline GSTT1 genotype & 12 & $1,696 / 1,542$ & $1.284[0.963-1.712]$ & 0.089 \\
\hline GSTM1+GSTT1 genotype & 3 & $458 / 320$ & 1.797 [1.081-2.989] & 0.024 \\
\hline \multicolumn{5}{|c|}{ Controls from healthy individuals } \\
\hline GSTM1 genotype & 12 & $1,391 / 2,200$ & $1.569[1.131-2.176]$ & 0.007 \\
\hline GSTT1 genotype & 6 & $675 / 948$ & $1.315[0.767-2.254]$ & 0.320 \\
\hline GSTM1+GSTT1 genotype & 5 & $625 / 902$ & $1.657[1.085-2.532]$ & 0.001 \\
\hline \multicolumn{5}{|l|}{ High quality } \\
\hline GSTM1 genotype & 23 & $2,920 / 3,426$ & $1.563[1.253-1.949]$ & $<0.001$ \\
\hline GSTT1 genotype & 14 & $1,898 / 1,841$ & $1.376[1.020-1.858]$ & 0.037 \\
\hline$G S T M 1+G S T T 1$ genotype & 6 & $854 / 878$ & $1.753[1.265-2.430]$ & 0.001 \\
\hline \multicolumn{5}{|l|}{ Low quality } \\
\hline GSTM1 genotype & 10 & $1,070 / 1,199$ & $1.259[0.785-2.020]$ & 0.340 \\
\hline GSTT1 genotype & 4 & $473 / 649$ & $1.121[0.646-1.944]$ & 0.684 \\
\hline$G S T M 1+G S T T 1$ genotype & 2 & $229 / 344$ & $1.542[1.009-2.356]$ & 0.045 \\
\hline
\end{tabular}

OR, odds ratio; CI, confidence interval; GSTM1, glutathione S-transferase $\mu 1$; GSTT1, glutathione S-transferase $\theta 1$.

association between the combined GSTM1/GSTT1 null genotype and an increased risk for endometriosis $(\mathrm{OR}=1.68$, 95\% CI: 1.29-2.17; P<0.0001; Fig. 2C).

This association was unchanged by subgroup analyses stratified by source of controls (hospital-based studies: $\mathrm{OR}=1.797 ; 95 \% \mathrm{CI}: 1.081-2.989 ; \mathrm{P}=0.024$; healthy individuals: $\mathrm{OR}=1.657 ; 95 \% \mathrm{CI}: 1.085-2.532 ; \mathrm{P}=0.001)$ or quality of evidence (high-quality evidence: $\mathrm{OR}=1.753$; 95\% CI: $1.265-2.430 ; \mathrm{P}=0.001$; low-quality evidence: $\mathrm{OR}=1.542$; $95 \%$ CI: 1.009-2.356, $\mathrm{P}=0.045$; Table II).

Subgroup analysis stratified by ethnicity demonstrated a significant association between the combined GSTM1/GSTT1 null genotype and an increased risk for endometriosis among Asian populations (OR=1.898; 95\% CI: 1.404-2.565; $\mathrm{P}<0.001)$, but not among Caucasian populations $(\mathrm{OR}=1.185$; 95\% CI: 0.717-1.961; $\mathrm{P}=0.508)$.
Publication bias. Visual inspection of a Funnel plot, Egger's test and Begg's rank correlation test revealed no significant publication bias for the GSTM1, GSTT1 and combined GSTM1/GSTT1 studies (Fig. 3; Table IV).

Heterogeneity analysis. There was evidence of significant heterogeneity $\left(\mathrm{I}^{2}>50 \%\right)$ between studies of GSTM1 and GSTT1, and those used in subgroup analyses, although not among studies of GSTM1/GSTT1 combined (Table IV). Therefore, the random-effect model was used in all analyses with the exception of the analysis of combined GSTM1/GSTT1 gene polymorphisms. For the GSTM1 and GSTT1 gene polymorphisms, a meta-regression was conducted in which publication year, ethnicity, source of controls, sample size, and quality of evidence were covariates. All the covariates were entered into the meta-regression model simultaneously, 
A

$$
\begin{aligned}
& \text { Study } \\
& \text { ID }
\end{aligned}
$$$$
\text { OR }(95 \% \mathrm{Cl}) \quad \text { Weight }
$$

Baranov VS (1996)

Baranov VS (1999)

Baranova H (1999)

Hadfield RM (2001)

Baxter SW (2001)

Bischoff FZ (2002)

Peng DX (2003)

Ivaschenko TE (2003)

Lin J (2003)

Arvanitis DA (2003)

De Carvalho CV (2004)

Morizane M (2004)

Hsieh YY (2004)

Ding $Y(2004)$

Ding $Y(2004)$

Hur SE (2005)

Babu KA (2005)

Kim SH (2007)

Chang Y (2007)

Aban M (2007)

Cao YH (2009)

Rozati R (2009)

Wu HL (2009)

Yang LP (2009)

Huang PC (2010)

Hosseinzadeh Z (2011)

Trabert B (2011)

$\mathrm{Wu} C \mathrm{CH}$ (2012)

Matsuzaka Y (2012)

Seifati SM (2012)

Vichi S (2012)

Frare AB (2013)

Sachan S (2013)

Overall ( $($-squared $=81.8 \%, p=0.000$ )

NOTE: Weights are from random effects analysis

$$
0142
$$

$6.70(2.72,16.43) \quad 2.36$

$1.93(1.15,3.22) \quad 3.27$

$3.94(1.89,8.21) \quad 2.75$

$0.75(0.40,1.42) \quad 2.97$

$0.95(0.58,1.57) \quad 3.29$

$0.21(0.09,0.52) \quad 2.40$

$2.23(1.17,4.25) \quad 2.96$

$1.78(0.82,3.84) \quad 2.66$

$3.44(1.39,8.51) \quad 2.36$

$1.29(0.94,1.77) \quad 3.67$

$0.46(0.20,1.10) \quad 2.45$

$1.05(0.65,1.70) \quad 3.34$

$32.60(15.07,70.32) \quad 2.64$

$0.92(0.45,1.88) \quad 2.79$

$1.23(0.69,2.20) \quad 3.10$

$1.07(0.74,1.56) \quad 3.56$

$1.51(1.04,2.19) \quad 3.57$

$1.04(0.74,1.45) \quad 3.64$

$2.15(1.09,4.25) \quad 2.88$

$1.86(1.18,2.93) \quad 3.39$

$1.23(0.62,2.46) \quad 2.84$

$\begin{array}{ll}2.12(1.05,4.28) & 2.82\end{array}$

$2.15(1.18,3.90) \quad 3.07$

$1.90(1.29,2.78) \quad 3.55$

$1.43(0.50,4.10) \quad 2.05$

$3.95(2.43,6.45) \quad 3.32$

$\begin{array}{ll}1.31(0.97,1.76) & 3.70\end{array}$

$2.04(1.26,3.30) \quad 3.34$

$0.90(0.54,1.51) \quad 3.26$

$0.94(0.56,1.56) \quad 3.28$

$1.22(0.80,1.87) \quad 3.46$

$0.35(0.15,0.83) \quad 2.48$

$3.63(1.77,7.46) \quad 2.78$

$1.56(1.25,1.95) \quad 100.00$

B

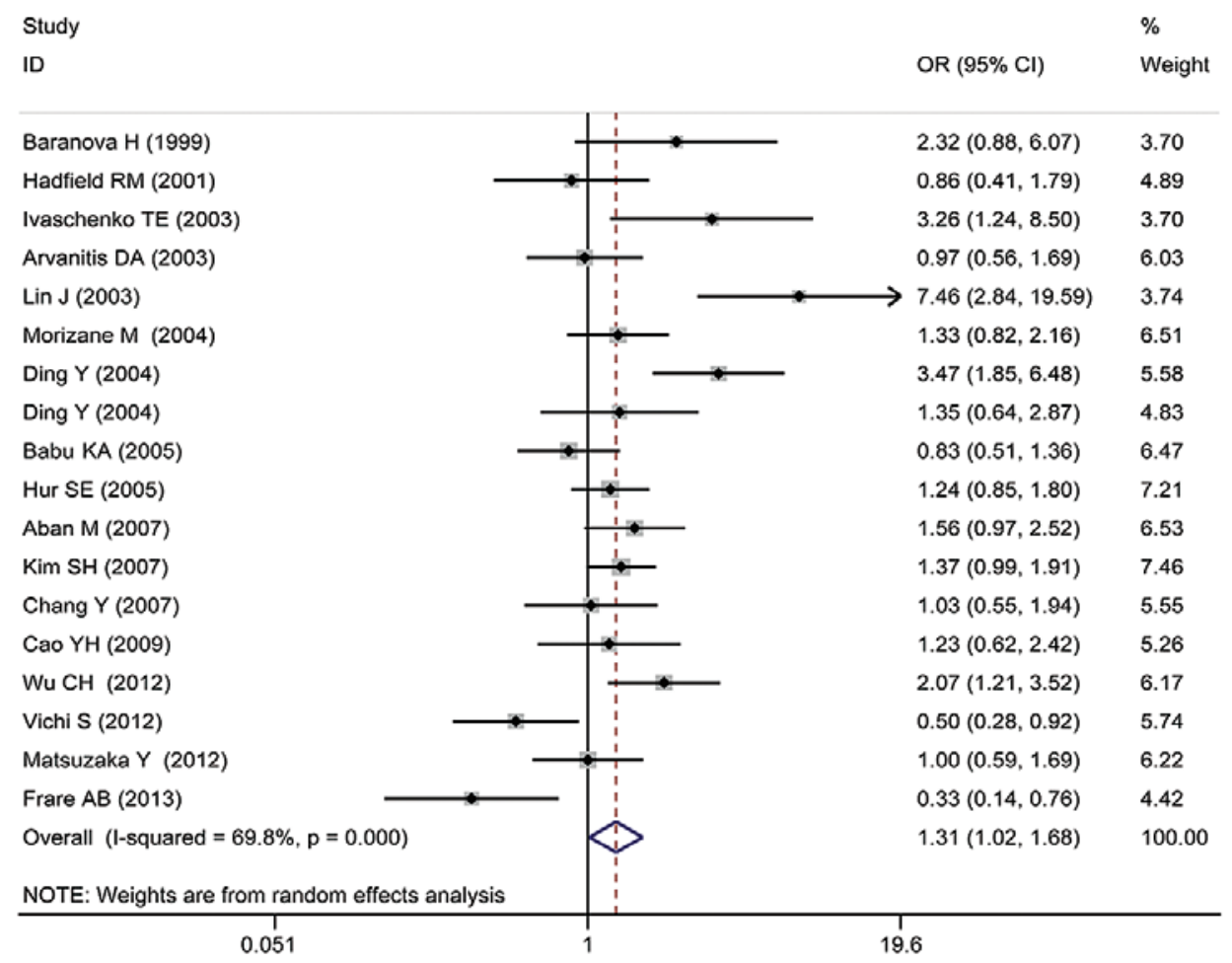

Figure 2. Association between GSTM1, GSTT1 and the combined GSTM1/GSTT1 null genotypes and susceptibility to endometriosis. (A) A total of 33 studies described the association between the GSTM1 null genotype and susceptibility to endometriosis [odds ratio (OR) $=1.56$; $95 \%$ confidence interval (CI): 1.25-1.95; P<0.0001]; and (B) 18 studies described the association between the GSTT1 null genotype and susceptibility to endometriosis (OR=1.31; 95\% CI: 1.02-1.68; $\mathrm{P}=0.037)$. GSTM1, glutathione S-transferase $\mu 1$;. GSTT1, glutathione S-transferase $\theta 1$. 


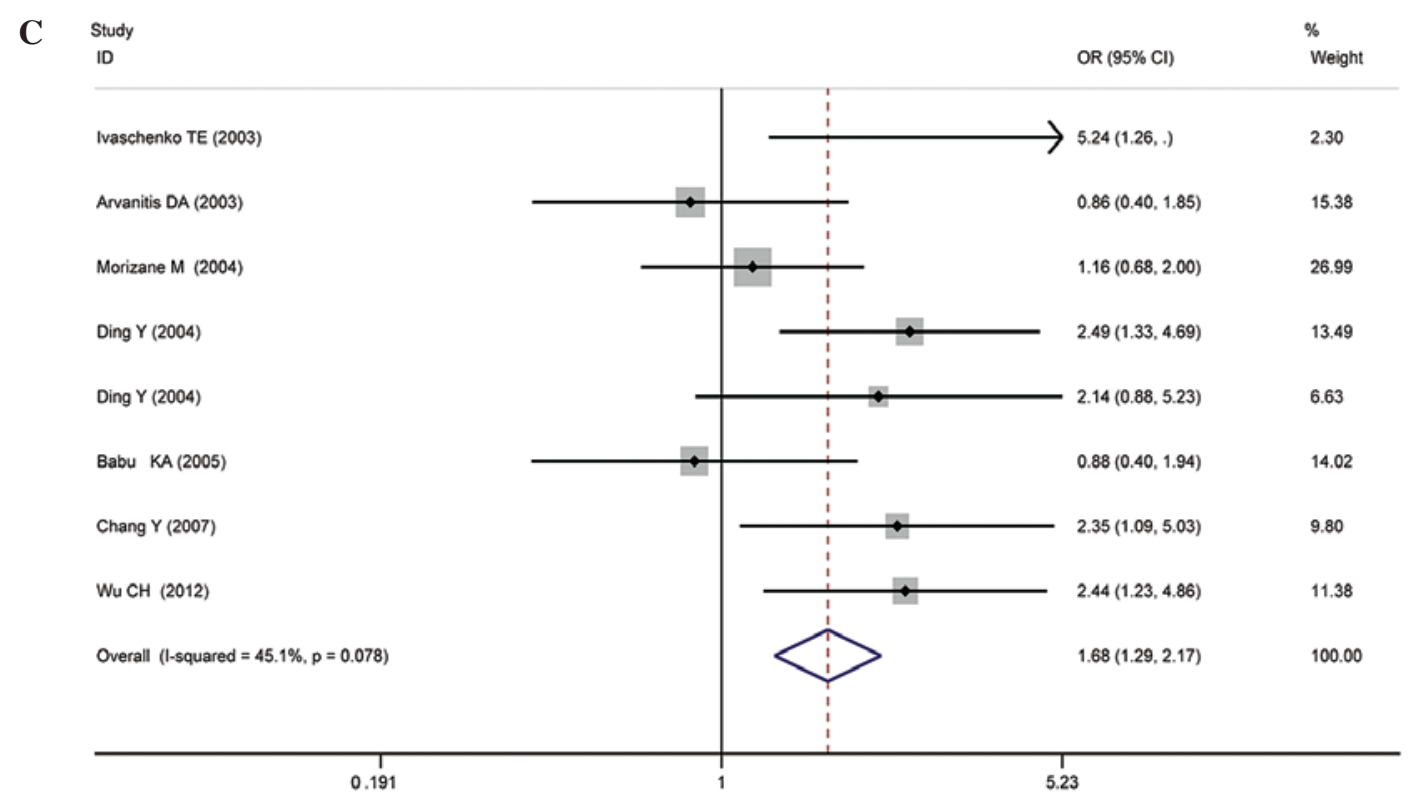

Figure 2. Continued. (C) Eight studies described the association between the combined GSTM1/GSTT1 null genotypes and susceptibility to endometriosis [odds ratio $(\mathrm{OR})=1.68 ; 95 \%$ confidence interval (CI): 1.29-2.17; P<0.0001]. GSTM1, glutathione S-transferase $\mu 1$; . GSTT1, glutathione S-transferase $\theta 1$.
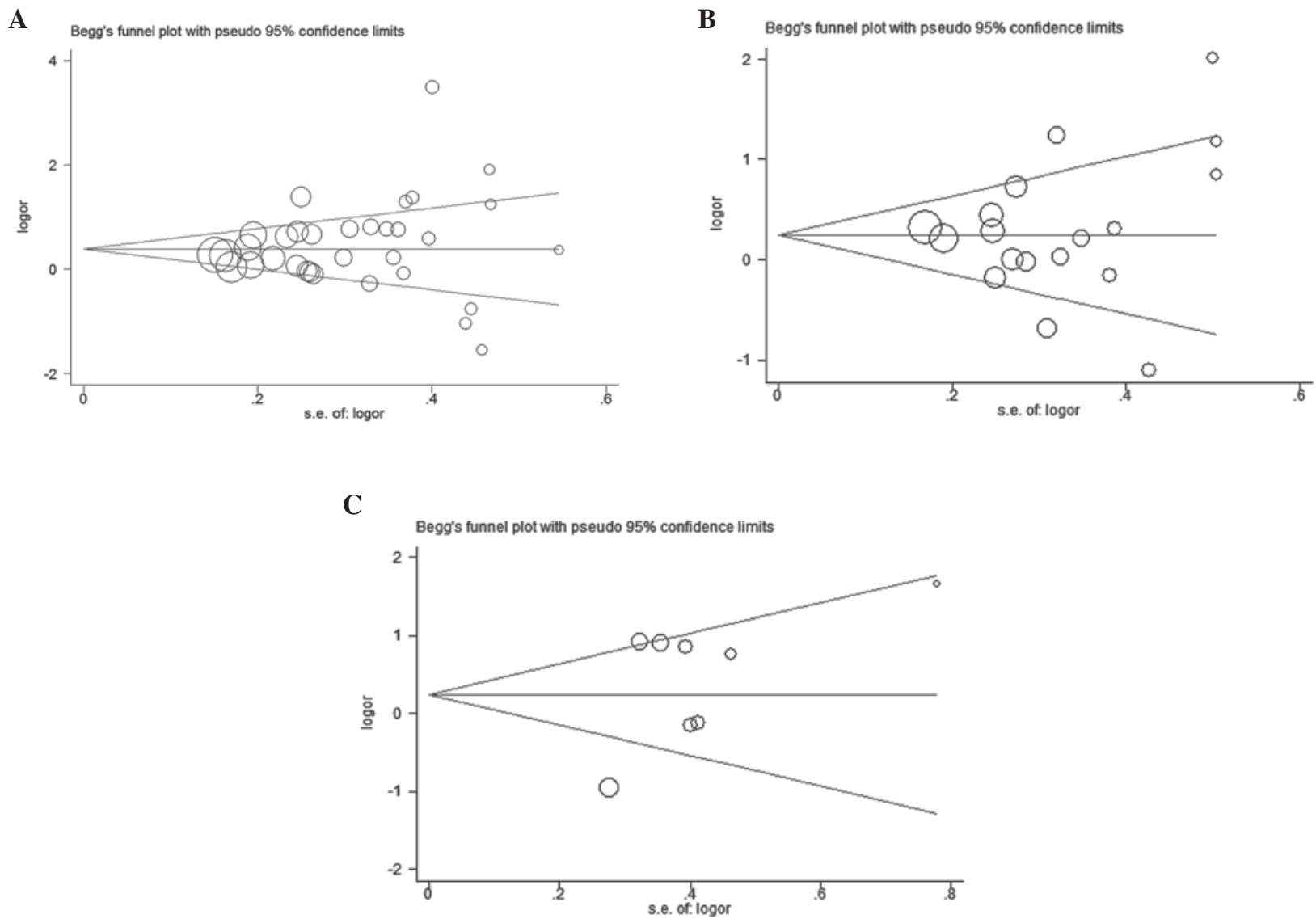

Figure 3. Assessment of publication bias for studies on (A) GSTM1, (B) GSTT1 and (C) combined GSTM1/GSTT1 genotypes. GSTM1, glutathione S-transferase $\mu 1 ;$. GSTT1, glutathione S-transferase $\theta 1$.

and the covariates that had the highest P-values were omitted one at a time in order to identify any sources of heterogeneity among them. However, the meta-regression analysis did not identify any of these covariates as a significant source of heterogeneity (Figs. 4 and 5).
Sensitivity analysis. To explore the effects of individual studies on the pooled OR estimates, a sensitivity analysis was performed, with the omission of one study at a time. The OR estimates for the GSTM1 polymorphism were not notably altered (Fig. 6A). The OR estimates for the GSTT1 and 
Table III. Comparisons of subgroup analyses for GSTM1, GSTT1 and combined GSTM1/GSTT1 studies.

A, Analysis of the GSTM1 gene

\begin{tabular}{|c|c|c|c|c|c|}
\hline \multirow[b]{2}{*}{ Subgroup } & \multirow[b]{2}{*}{ Subjects } & \multicolumn{2}{|c|}{ GSTMI (n) } & \multirow[b]{2}{*}{$\chi^{2}$} & \multirow[b]{2}{*}{ P-value } \\
\hline & & Null & Normal & & \\
\hline \multicolumn{4}{|l|}{ Ethnicity } & 3.245 & $0.72^{\mathrm{a}}$ \\
\hline \multirow[t]{2}{*}{ Caucasian } & Cases & 1,128 & 1,035 & & \\
\hline & Controls & 1,120 & 1,449 & & \\
\hline \multirow[t]{2}{*}{ Asian } & Cases & 1,003 & 713 & & \\
\hline & Controls & 909 & 1,069 & & \\
\hline \multirow[t]{2}{*}{ Mixed } & Cases & 46 & 65 & 18.737 & $<0.001^{\mathrm{b}}$ \\
\hline & Controls & 51 & 27 & 23.467 & $<0.001^{\mathrm{c}}$ \\
\hline \multicolumn{4}{|l|}{ Source of controls } & 0.130 & 0.718 \\
\hline \multirow[t]{2}{*}{ Hospital patients } & Cases & 1,397 & 1,202 & & \\
\hline & Controls & 1,073 & 1,352 & & \\
\hline \multirow[t]{2}{*}{ Healthy individuals } & Cases & 780 & 611 & & \\
\hline & Controls & 1,007 & 1,193 & & \\
\hline \multirow{3}{*}{$\begin{array}{l}\text { Quality } \\
\text { High quality }\end{array}$} & & & & 0.825 & 0.364 \\
\hline & Cases & 1,609 & 1,311 & & \\
\hline & Controls & 1,539 & 1,887 & & \\
\hline \multirow[t]{2}{*}{ Low quality } & Cases & 568 & 502 & & \\
\hline & Controls & 541 & 658 & & \\
\hline
\end{tabular}

B, Analysis of the GSTT1 gene

\begin{tabular}{|c|c|c|c|c|c|}
\hline \multirow[b]{2}{*}{ Subgroup } & \multirow[b]{2}{*}{ Subjects } & \multicolumn{2}{|c|}{ GSTT1 (n) } & \multirow[b]{2}{*}{$\chi^{2}$} & \multirow[b]{2}{*}{ P-value } \\
\hline & & Null & Normal & & \\
\hline \multicolumn{4}{|l|}{ Ethnicity } & 6.766 & 0.009 \\
\hline \multirow[t]{2}{*}{ Caucasian } & Cases & 214 & 957 & & \\
\hline & Controls & 168 & 867 & & \\
\hline \multirow[t]{2}{*}{ Asian } & Cases & 607 & 543 & & \\
\hline & Controls & 568 & 841 & & \\
\hline Source of controls & & & & 0.638 & 0.425 \\
\hline \multirow[t]{2}{*}{ Hospital patients } & Cases & 631 & 1,065 & & \\
\hline & Controls & 522 & 1,020 & & \\
\hline \multirow[t]{2}{*}{ Healthy individuals } & Cases & 206 & 469 & & \\
\hline & Controls & 241 & 707 & & \\
\hline Quality & & & & 0.062 & 0.803 \\
\hline \multirow[t]{2}{*}{ High quality } & Cases & 625 & 1,273 & & \\
\hline & Controls & 507 & 1,334 & & \\
\hline \multirow[t]{2}{*}{ Low quality } & Cases & 212 & 261 & & \\
\hline & Controls & 256 & 393 & & \\
\hline
\end{tabular}

C, Analysis of GSTM1+GSTT1 genes

\begin{tabular}{lllllr}
\hline & & \multicolumn{2}{c}{ GSTMI+GSTT1 (n) } & & \\
\cline { 5 - 6 } Subgroup & Subjects & Null & Normal & $\chi^{2}$ & P-value \\
\hline Ethnicity & & & & 7.642 & 0.006 \\
Caucasian & Cases & 41 & 618 & &
\end{tabular}


Table III. Continued.

\begin{tabular}{llccc}
\hline Subgroup & Subjects & Null & Normal & $\chi^{2}$ \\
\hline Asian & Cases & 124 & 300 & P-value \\
Cource of controls & Controls & 109 & 152 & 0.091 \\
Hospital patients & Cases & 57 & 401 & 0.763 \\
& Controls & 26 & 294 & 0.022 \\
Healthy individuals & Cases & 108 & 517 & 0.882 \\
Quality & Controls & 112 & 790 & \\
High quality & & & 542 & 598 \\
Low quality & Cases & 112 & 176 & \\
& Controls & 80 & 286 & \\
\hline
\end{tabular}

${ }^{\mathrm{a} C}$ Caucasians vs. Asians, ${ }^{\mathrm{b}}$ Caucasians vs. mixed, ${ }^{\mathrm{c}}$ Asians vs. mixed.

Table IV. Heterogeneity and publication bias of GSTM1, GSTT1 and combined GSTM1/GSTT1 studies.

\begin{tabular}{|c|c|c|c|c|}
\hline \multirow[b]{2}{*}{ Group } & \multicolumn{2}{|c|}{ Heterogeneity } & \multicolumn{2}{|c|}{ Publication bias (P-value) } \\
\hline & $\mathrm{I}^{2}$ value $(\%)$ & P-value & Egger's test & Begg's funnel plot \\
\hline \multicolumn{5}{|l|}{ Total studies } \\
\hline GSTM1 genotype & 81.8 & $<0.001$ & 0.313 & 0.412 \\
\hline GSTT1 genotype & 69.9 & $<0.001$ & 0.557 & 0.705 \\
\hline GSTM1+GSTT1 genotype & 44.7 & 0.081 & 0.170 & 1.000 \\
\hline \multicolumn{5}{|l|}{ Caucasian } \\
\hline GSTM1 genotype & 79.2 & $<0.001$ & 0.454 & 0.322 \\
\hline GSTT1 genotype & 64.7 & 0.009 & 0.339 & 0.764 \\
\hline GSTM1+GSTT1 genotype & 58.5 & 0.090 & 0.021 & 0.296 \\
\hline \multicolumn{5}{|l|}{ Asian } \\
\hline GSTM1 genotype & 83.8 & $<0.001$ & 0.098 & 0.083 \\
\hline GSTT1 genotype & 62.4 & 0.004 & 0.160 & 0.210 \\
\hline GSTM1+GSTT1 genotype & 13.6 & 0.081 & 0.340 & 0.806 \\
\hline \multicolumn{5}{|l|}{ Mixed } \\
\hline GSTM1 genotype & 0.0 & 0.664 & $<0.001$ & 0.317 \\
\hline \multicolumn{5}{|l|}{ Controls from hospital patients } \\
\hline GSTM1 genotype & 83.6 & $<0.001$ & 0.390 & 0.506 \\
\hline GSTT1 genotype & 65.9 & 0.001 & 0.335 & 0.451 \\
\hline$G S T M 1+G S T T 1$ genotype & 62.2 & 0.071 & 0.585 & 1.000 \\
\hline \multicolumn{5}{|c|}{ Controls from healthy individuals } \\
\hline GSTM1 genotype & 79.4 & $<0.001$ & 0.598 & 0.784 \\
\hline GSTT1 genotype & 78.4 & $<0.001$ & 0.431 & 0.707 \\
\hline GSTM1+GSTT1 genotype & 45.9 & 0.116 & 0.531 & 1.000 \\
\hline \multicolumn{5}{|l|}{ High quality } \\
\hline GSTM1 genotype & 80.9 & $<0.001$ & 0.042 & 0.068 \\
\hline GSTT1 genotype & 69.9 & $<0.001$ & 0.530 & 0.189 \\
\hline GSTM1+GSTT1 genotype & 49.0 & 0.081 & 0.641 & 1.000 \\
\hline \multicolumn{5}{|l|}{ Low quality } \\
\hline GSTM1 genotype & 85.1 & $<0.001$ & 0.788 & 0.516 \\
\hline GSTT1 genotype & 77.2 & 0.004 & 0.347 & 1.000 \\
\hline GSTM1+GSTT1 genotype & 62.9 & 0.101 & & \\
\hline
\end{tabular}

GSTM1, glutathione S-transferase $\mu 1$;. GSTT1, glutathione S-transferase $\theta 1$. 
A

\begin{tabular}{|c|c|c|c|c|c|c|}
\hline \multicolumn{5}{|c|}{$\begin{array}{l}\text { Meta-regression } \\
\text { Rba. estimate of betveen-study variance } \\
\text { \& residual variation due to heterogeneity } \\
\text { proportion of between-gtudy variance explained } \\
\text { Joint test for all covariates } \\
\text { Hith Knapp-Hartung modisication }\end{array}$} & $\begin{array}{l}\text { Number of obs } \\
\text { tauz } \\
\text { I-squared_res } \\
\text { ldj } R \text {-squared } \\
\text { Mode1 } r(5,27) \\
\text { Prob }>r\end{array}$ & $\begin{array}{r}=\quad 33 \\
=\quad .658 \\
=84.156 \\
=-17.930 \\
=0.34 \\
=0.8865\end{array}$ \\
\hline logor & coes. & Sta. Err. & $\tau$ & $P>|C|$ & [95t cone. & Interval] \\
\hline publish & -.0222449 & .0523803 & -0.42 & 0.674 & -.1297205 & .0852307 \\
\hline gize & -.0057332 & .0930674 & -0.06 & 0.951 & -.1966917 & .1852253 \\
\hline ethnieity & -.1694732 & .2954847 & -0.57 & 0.571 & -.7757577 & .4368114 \\
\hline source & -.1049237 & .3379029 & -0.31 & 0.759 & -.7982432 & .5883958 \\
\hline quality & .2929028 & .362554 & 0.81 & 0.426 & -.4509965 & 1.036802 \\
\hline _cons & .5558104 & 1.054713 & 0.53 & 0.603 & -1.608282 & 2.719903 \\
\hline
\end{tabular}

B

\begin{tabular}{|c|c|c|c|c|c|c|}
\hline & \multicolumn{6}{|c|}{ - metaxeg logor publish ethnicity source guality, vsse(_selogES) bsest(xeml) } \\
\hline \multirow{6}{*}{\multicolumn{5}{|c|}{$\begin{array}{l}\text { Meta-regression } \\
\text { RDa. estibate of between-study variance } \\
\text { s residual variation due to heterogeneity } \\
\text { Proportion of betueen-study variance explained } \\
\text { Joint test sor all covariates } \\
\text { Hith Knapp-Hartung wodification }\end{array}$}} & Nurber of obs & 33 \\
\hline & & & & & tauz & .6263 \\
\hline & & & & & I-squared_res & $83.59 \mathrm{t}$ \\
\hline & & & & & Ad) R-squared & $=-12.25 \theta$ \\
\hline & & & & & Hode1 $F(4,28)$ & $=0.43$ \\
\hline & & & & & Prob $>F$ & 0.7846 \\
\hline $1000 \mathrm{r}$ & coer. & Sed. Err. & $\mathrm{e}$ & $P>|E|$ & [9S: Cons. & Interval] \\
\hline publish & -.0228103 & .0501709 & -0.45 & 0.653 & -.1255807 & .07996 \\
\hline echniciey & -.1629092 & .2725722 & -0.60 & 0.555 & -.7212481 & .3954297 \\
\hline source & -.1010562 & .3256125 & -0.31 & 0.759 & -.7680431 & .5659308 \\
\hline qualicy & .2884664 & .3536127 & 0.82 & 0.422 & -.4358765 & 1.012809 \\
\hline cons & .5321084 & .9521008 & 0.56 & 0.581 & -1.418182 & 2.482399 \\
\hline
\end{tabular}

C

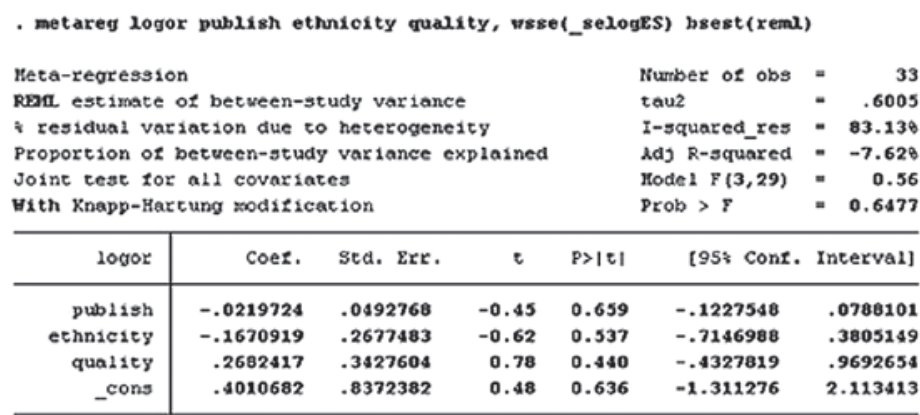

D

metareg logor ethnicity quality, rsse(_selogES) bsest(remu)

Heta-regression

Rad estimate or between-study variance

* residual variation due to heterogeneity

Proportion or betveen-scudy variance explained

Joint test for all covariates

Hith Knapp-Hartung modification

\begin{tabular}{|c|c|c|c|c|c|c|}
\hline logor & coer. & sta. Err. & $t$ & $P>|t|$ & (95t Cons. & Interval] \\
\hline ethniesty & -.1886307 & .259498 & -0.73 & 0.473 & -.7185963 & .341335 \\
\hline quality & .2568094 & .3371358 & 0.76 & 0.452 & -.4317137 & .9453325 \\
\hline _cons & .30802 & .798419 & 0.39 & 0.702 & -1.322569 & 1. 938609 \\
\hline
\end{tabular}

$\mathbf{E}$

\begin{tabular}{|c|c|c|c|c|c|c|}
\hline \multicolumn{5}{|c|}{$\begin{array}{l}\text { Ieta-regression } \\
\text { Raal estimate of betveen-study variance } \\
\text { i residual vatiation due to heterogeneity } \\
\text { proportion of betveen-study variance explained } \\
\text { Hith Knapp-Hartung modification }\end{array}$} & $\begin{array}{l}\text { Number of obs } \\
\text { tau2 } \\
\text { I-squared_res } \\
\text { ldj R-squared }\end{array}$ & $\begin{array}{rr}= & 33 \\
= & .5648 \\
= & 82.238 \\
= & -1.238\end{array}$ \\
\hline logor & Coef. & Std. Err. & $t$ & $P>|t|$ & [9S: Cont. & Interval) \\
\hline $\begin{array}{r}\text { quality } \\
\text { cons }\end{array}$ & .3201558 & .3226287 & 0.99 & 0.329 & -.3378498 & $\begin{array}{r}.9781615 \\
1.065436\end{array}$ \\
\hline & . & .0000129 & & 0.803 & -1.2500190 & \\
\hline
\end{tabular}

Number of obs $=33$

tau2 $=.5775$

I-squared_res $=82.560$ Hode $1 F(2,30)=0.75$ Prob $>F(2,30)=0.480$

[rval] 
A

. metareg logor quality ethnioity source publish size, vrse(_selogEs) bsest(reml)

Heta-regression

REII. estimate of betueen-study variance

; residual variation due to heterogeneity

proportion of betueen-study variance explained

Joint test for all covariates

Hith Knapp-Hartung rodification

$\begin{array}{llr}\text { Number of obs } & = \\ \text { tau2 } & =28 \\ \text { I-squared_res } & =21.886 \\ \text { ldj } R-3 q u a r e d & =-6.388 \\ \text { Hode1 } F(5,12) & =1.16 \\ \text { Prob }>F & =0.3826\end{array}$

\begin{tabular}{r|rrrrrr}
\hline logor & Coef. & Std. Err. & $\tau$ & P $>|\tau|$ & [9Si Cont. Interval] \\
\hline quality & -.1590068 & .4528592 & -0.35 & 0.732 & -1.145702 & .8276887 \\
ethnicity & .271699 & .4052781 & 0.67 & 0.515 & -.6113262 & 1.154724 \\
source & -.0803778 & .3782303 & -0.21 & 0.835 & -.9044707 & .7437151 \\
publish & -.1704023 & .0853161 & -2.00 & 0.069 & -.3562901 & .0154854 \\
Size & -.0645026 & .098486 & -0.65 & 0.525 & -.2790851 & .1500799 \\
_cons & 1.239194 & .7636197 & 1.62 & 0.131 & -.4245906 & 2.902978 \\
\hline
\end{tabular}

B

. metareg logor guality ethnioxty publish size, vsse(_selogES) bsest(remd)

Jeta-regression

peall estimate of betueen-study variance

* residual variation due to heterogeneity

proportion or betveen-study variance explained

Joint test for all covariates

With Knapp-Hartung rodification

Nurber of obs $=18$

rauz $\quad .2512$

$1-5 q$ uared_res -69.500

(d) $\mathrm{R}$-squared $=3.39$

Prob $>r=0.2518$

\begin{tabular}{r|rrrrrr}
\hline logor & Coef. & Std. Err. & $t$ & $\mathrm{P}>|\mathrm{t}|$ & \multicolumn{2}{c}{ [95t Conf. Interval] } \\
\hline quality & -.1857158 & .4081106 & -0.46 & 0.657 & -1.067385 & .6959535 \\
ethnicity & .250042 & .3759013 & 0.67 & 0.518 & -.5620434 & 1.062127 \\
publish & -.1645894 & .0793835 & -2.07 & 0.059 & -.3360871 & .0069084 \\
size & -.0651066 & .0939738 & -0.69 & 0.501 & -.2681247 & .1379114 \\
cons & 1.173268 & .6945433 & 1.69 & 0.115 & -.3272015 & 2.673738 \\
\hline
\end{tabular}

C

- metareg logor ethnioity publish size, wsse(_selogES) bsest(reml)

Meta-regression

REal estirate of betveen-study variance

i residual variation due to heterogeneity

Proportion of between-study variance explained

Joint test for all covariates

With Knapp-Hartung modification

$\begin{array}{llr}\text { Number of obs } & =18 \\ \text { tau2 } & = & -2216 \\ \text { I-squared_res } & =67.698 \\ \text { Adj R-squared } & =18.33 \\ \text { Mode1 F }(3,14) & =2.05 \\ \text { Prob }>\text { F } & =0.1527\end{array}$

\begin{tabular}{r|rrrrrr}
\hline logor & Coef. & Std. Err. & $t$ & P $>|t|$ & [95: Conf. Interval] \\
\hline ethnicity & .1674779 & .3162035 & 0.53 & 0.605 & -.5107112 & .845667 \\
publish & -.1629358 & .0767844 & -2.12 & 0.052 & -.3276219 & .0017504 \\
size & -.0722779 & .0891182 & -0.81 & 0.431 & -.2634175 & .1188617 \\
_cons & 1.097336 & .6596905 & 1.66 & 0.118 & -.3175592 & 2.512232 \\
\hline
\end{tabular}

D

- metareg logor publish size, vsse(_selogES) bsest(reml)

Meta-regression

REII estimate of between-study variance

* residual variation due to heterogeneity

Proportion of between-study variance explained

Joint test for all covariates

With Knapp-Hartung modification

$\begin{array}{lrr}\text { Number of obs } & =18 \\ \text { tau2 } & = & 12043 \\ \text { I-squared_res } & =66.880 \\ \text { Adj R-squared } & =24.688 \\ \text { Model F }(2,15) & =3.05 \\ \text { Prob }>F & =0.0773\end{array}$

\begin{tabular}{r|rrrrrr}
\hline logor & Coef. & Std. Err. & $t$ & P $>|t|$ & [95: Conf. Interval] \\
\hline publish & -.1420633 & .0653113 & -2.18 & 0.046 & -.2812709 & -.0028556 \\
size & -.0925823 & .0779051 & -1.19 & 0.253 & -.2586331 & .0734686 \\
- cons & 1.337196 & .4586958 & 2.92 & 0.011 & .3595093 & 2.314883 \\
\hline
\end{tabular}

E

\begin{tabular}{|c|c|c|c|c|c|c|}
\hline \multicolumn{5}{|c|}{$\begin{array}{l}\text { - metareg logor publish, wsse(_selogES) bsest (reml) } \\
\text { Heta-regression } \\
\text { Read estimate of betveen-study variance } \\
\text { \& residual variation due to heterogeneity } \\
\text { Proportion of between-study variance explained } \\
\text { With Knapp-Hartung modification }\end{array}$} & $\begin{array}{l}\text { Number of obs } \\
\text { tau2 } \\
\text { I-squared res } \\
\text { kdj k-squared }\end{array}$ & $\begin{array}{rr}= & 18 \\
= & .2125 \\
= & 67.218 \\
= & =-21.678\end{array}$ \\
\hline logor & Coef. & Std. Err. & $t$ & $P>|t|$ & [95* Cons. & Interval] \\
\hline publish & -.141961 & .0661497 & -2.15 & 0.048 & -.2821921 & -.0017298 \\
\hline _cons & 1.020376 & .3760155 & 2.71 & 0.015 & $.223258 d$ & 1.817493 \\
\hline
\end{tabular}

Figure 5. Meta-regression for GSTT1 studies, with publication year, ethnicity, source of controls, sample size, and quality of evidence as covariates. All covariates were entered into the meta-regression model simultaneously, and covariates with the highest P-values were omitted one at a time to identify sources of heterogeneity. Meta-regression identified publication year as a significant source of heterogeneity $(\mathrm{P}=0.048)$, but after omitting this covariate heterogeneity remained substantial $\left(I^{2}=67.21 \%\right)$ Variables were omitted in the order: Source $(A \rightarrow B)$, quality $(B \rightarrow C)$, ethnicity $(C \rightarrow D)$, size $(D \rightarrow E)$. GSTT1, glutathione S-transferase $\theta 1$. 
A

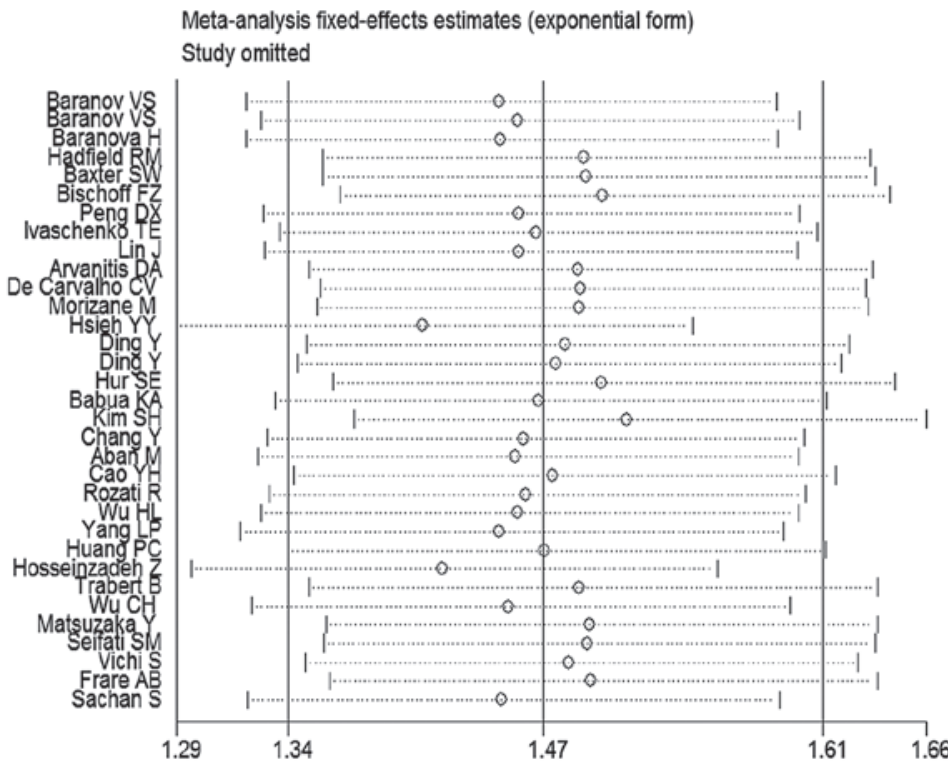

B

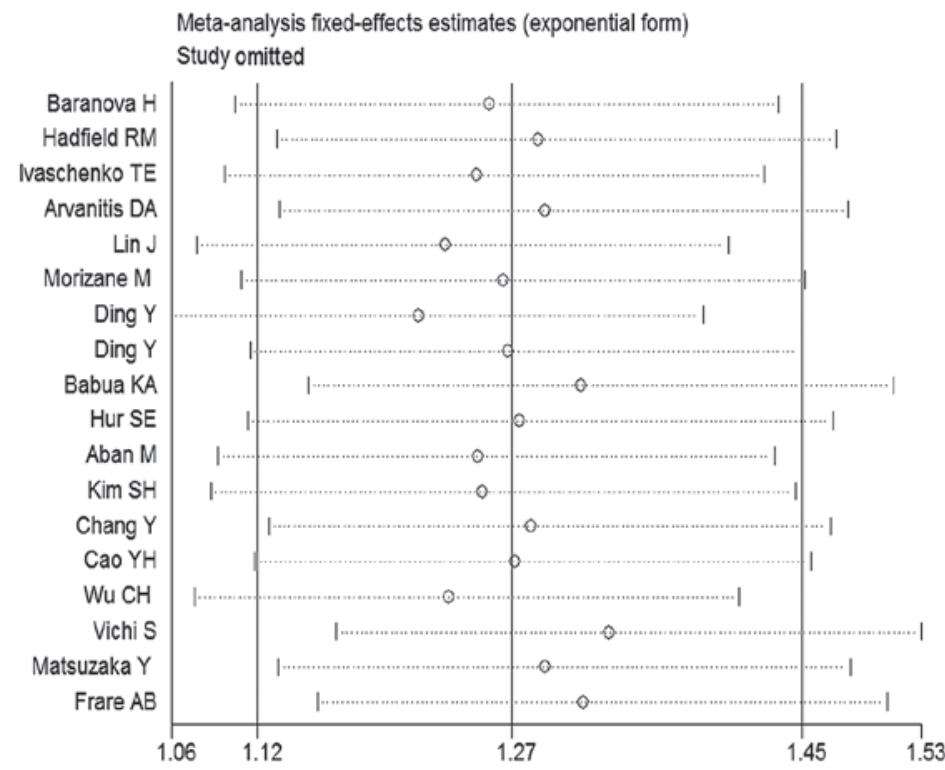

C

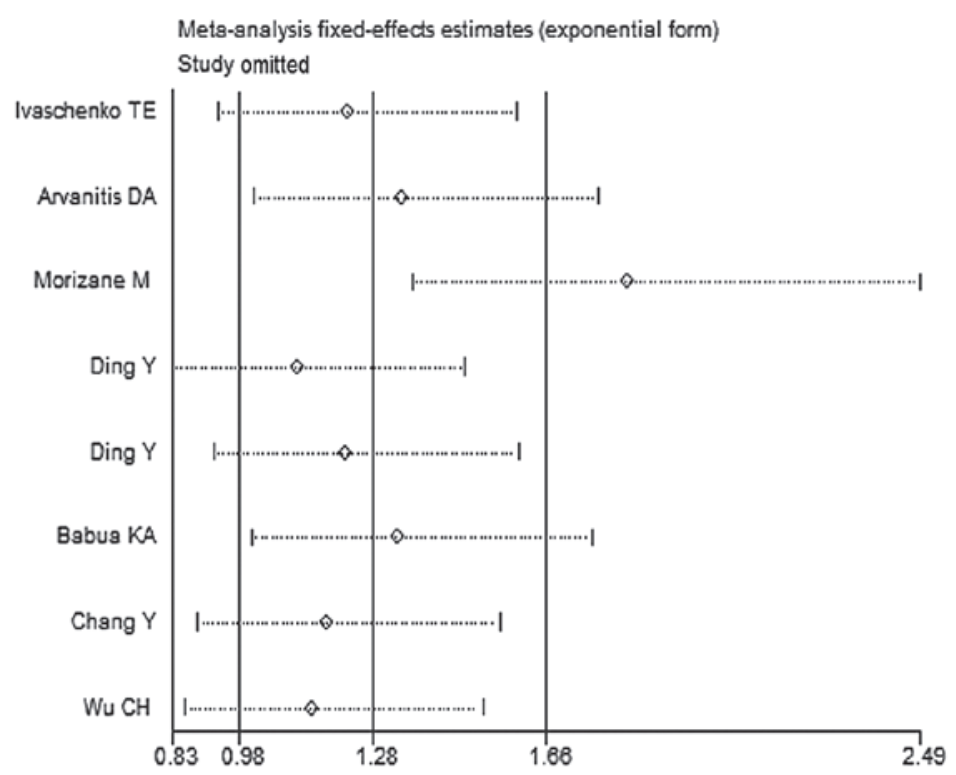

Figure 6. Sensitivity analyses investigating the association between the (A) GSTM1, (B) GSTT1 and (C) combined GSTM1/GSTT1 null genotypes and susceptibility to endometriosis; one study was omitted at a time. GSTM1, glutathione S-transferase $\mu 1$;. GSTT1, glutathione S-transferase $\theta 1$. 
combined GSTM1/GSTT1 polymorphisms were altered when studies were excluded (Fig. 6B and C).

\section{Discussion}

In the present study, a meta-analysis of data from 33 studies was conducted to examine the associations between the GSTM1, GSTT1 and combined GSTM1/GSTT1 null genotypes and susceptibility to endometriosis. The risk for endometriosis was significantly increased in the presence of the GSTM1, GSTT1 and combined GSTM1/GSTT1 null genotypes compared with the wild-type. Subgroup analyses stratified by ethnicity, source of controls and quality of evidence confirmed this finding among several subgroups, but particularly among studies considered high-quality evidence. Notably, among patients of mixed ethnicity, the GSTM1 null genotype was significantly associated with a decreased risk for endometriosis compared with the wild-type.

A similar meta-analysis of 23 studies performed in 2005 demonstrated an increased risk for endometriosis in women with the GSTT1 null genotype (8). However, the authors requested that their findings be interpreted with caution as asymmetry in the funnel plot was evident, which was likely due to publication bias (8). This previous study did not include subgroup analyses or an evaluation of the combined GSTM1/GSTT1 null genotype-endometriosis association.

Previous meta-analyses have found that the GSTM1/GSTT1 gene polymorphism is associated with cervical cancer (45), breast cancer $(46)$, bladder cancer $(47)$, gastric cancer $(48,49)$ and acute leukemia (50). In accordance with the observations of the present study, several studies have shown that the GSTM1 (OR=32.6, 95\% CI: 15.07-70.32, $\mathrm{P}<0.0001)(18)$ and GSTT1 (OR $>3 ; \mathrm{P}<0.0001)$ null genotypes $(12,16)$ are associated with an increased risk for endometriosis. However, other reports suggest the GSTM1 (OR=0.21, 95\% CI: 0.09-0.52, $\mathrm{P}<0.0001 ; \mathrm{OR}=0.35,95 \%$ CI: $0.15-0.83, \mathrm{P}<0.0001)(40,43)$, GSTT1 (OR $\geq 5 ; \mathrm{P}<0.0001)(16)$ and combined GSTM1/GSTT1 $(\mathrm{OR}=0.38 ; \mathrm{P}<0.001)(17)$ null genotypes are associated with a decreased risk for endometriosis. These divergent results may be explained by differences in GSTM1/GSTT1 null genotype frequencies and study locations. The frequency of the GSTM1/GSTT1 null genotype may vary from 10 to $65 \%$ depending on the region and population studied (51). Different study locations may introduce confounding variables associated with variations in lifestyles and exposures to toxic substances of the study populations.

The results of the present study must be interpreted with caution due to the presence of substantial heterogeneity. Among analyses of the studies of GSTM1 and GSTT1, the cause of heterogeneity remains unclear, despite meta-regression analyses being conducted. Among the analyses of combined GSTM1/GSTT1 studies, subgroup and sensitivity analyses suggested that studies that included patients with advanced stage endometriosis caused most of the variability. Publication bias was unlikely to have influenced the findings.

In addition to the heterogeneity, there were several limitations to this study. Firstly, the composition of the endometriosis patient and control populations varied between studies. For instance, some studies included only patients with advanced endometriosis $(17-20,22,27,35)$, while control populations consisted of a mixture of infertile (29), postmenopausal (43) and premenopausal $(18,35)$ women, and newborn babies that had not been exposed to the environment (17). Furthermore, patients and controls were not always accurately matched by age or environmental exposures. Secondly, gene-gene or gene-environment interactions may jointly increase the risk for endometriosis; therefore, different lifestyle and environmental factors may contribute to differential genotypic frequencies in cases and controls. Attempts were made to mitigate inaccuracies associated with this limitation through a subgroup analysis stratified according to ethnicity. Thirdly, this study was based on published articles. As a positive result is more likely to be published, publication bias is an inherent limitation of all meta-analyses irrespective of the outcomes of the Egger's linear regression test and Begg's rank correlation test.

In conclusion, the present meta-analysis shows the GSTM1, GSTT1 and combined GSTM1/GSTT1 null genotypes are likely associated with increased susceptibility to endometriosis. These data are in contrast to those reported previously. Therefore, further studies reporting higher quality evidence are necessary to verify these conclusions.

\section{Acknowledgements}

The study was supported by Hubei Provincial Natural Science Foundation of China (Grant No.2013060602010236).

\section{References}

1. Viganò P, Parazzini F, Somigliana E and Vercellini P: Endometriosis: Epidemiology and aetiological factors. Best Pract Res Clin Obstet Gynaecol 18: 177-200, 2004.

2. Giudice LC, Tazuke SI and Swiersz L: Status of current research on endometriosis. J Reprod Med 43 (Suppl): 252-262, 1998.

3. Kennedy S: Is there a genetic basis to endometriosis? Semin Reprod Endocrinol 15: 309-318, 1997.

4. Mayani A, Barel S, Soback S and Almagor M: Dioxin concentrations in women with endometriosis. Hum Reprod 12: 373-375, 1997.

5. Eskenazi B, Mocarelli P, Warner M, Samuels S, Vercellini P, Olive D, Needham LL, Patterson DG Jr, Brambilla P, Gavoni N, et al: Serum dioxin concentrations and endometriosis: A cohort study in Seveso, Italy. Environ Health Perspect 110: 629-634, 2002.

6. Heilier JF, Nackers F, Verougstraete V, Tonglet R, Lison D and Donnez J: Increased dioxin-like compounds in the serum of women with peritoneal endometriosis and deep endometriotic (adenomyotic) nodules. Fertil Steril 84: 305-312, 2005.

7. Nebert DW and Vasiliou V: Analysis of the glutathione S-transferase (GST) gene family. Hum Genomics 1: 460-464, 2004.

8. Guo SW: Glutathione S-transferases M1/T1 gene polymorphisms and endometriosis: A meta-analysis of genetic association studies. Mol Hum Reprod 11: 729-743, 2005.

9. Guo SW: The association of endometriosis risk and genetic polymorphisms involving dioxin detoxification enzymes: A systematic review. Eur J Obstet Gynecol Reprod Biol 124: 134-143, 2006.

10. Hudelist G, Keckstein J and Wright JT: The migrating adenomyoma: Past views on the etiology of adenomyosis and endometriosis. Fertil Steril 92: 1536-1543, 2009.

11. Higgins JP and Thompson SG: Quantifying heterogeneity in a meta-analysis. Stat Med 21: 1539-1558, 2002.

12. Ding Y, Chen ZF, Lin RY, Wang XF, Ding JB, Ai XZ and Wen H: Relationship between endometriosis and glutathione S-transferase M1, T1 genes of the Uygurs and Hans in Xinjiang. Zhonghua Fu Chan Ke Za Zhi 39: 101-104, 2004 (In Chinese).

13. Rozati R, Baludu GS and Reddy BS: Possible aggravating impact of gene polymorphism in women with endometriosis. Indian J Med Res 129: 395-400, 2009. 
14. Yang LP and An XF: Analysis on the relationship between polymorphism of Exon7 situs in CYP1A1 gene, GSTM1 gene and susceptibility of endometriosis in women of Han nationality in Jilin City. Zhongguo Fu You Bao Jian 24: 2556-2564, 2009 (In Chinese)

15. Peng DX, He YL, Qiu LW, Yang F and Lin JM: Association between glutathione S-transferase M1 gene deletion and genetic susceptibility to endometriosis. Di Yi Jun Yi Da Xue Xue Bao 23: 458-459, 462, 2003 (In Chinese)

16. Lin J, Zhang X, Qian Y, et al: Glutathione S-transferase M1 and T1 genotypes and endometriosis risk: A case-controlled study. Chin Med J (Engl) 116: 777-780, 2003.

17. Morizane M, Yoshida S, Nakago S, Hamana S, Maruo T and Kennedy S: No association of endometriosis with glutathione S-transferase M1 and T1 null mutations in a Japanese population. J Soc Gynecol Investig 11: 118-121, 2004.

18. Hsieh YY, Chang CC, Tsai FJ, Lin CC, Chen JM and Tsai CH: Glutathione S-transferase M1*null genotype but not myeloperoxidase promoter G-463A polymorphism is associated with higher susceptibility to endometriosis. Mol Hum Reprod 10: 713-717, 2004.

19. Babu KA, Reddy NG, Deendayal M, Kennedy S and Shivaji S: GSTM1, GSTT1 and CYP1A1 detoxification gene polymorphisms and their relationship with advanced stages of endometriosis in South Indian women. Pharmacogenet Genomics 15: 167-172, 2005

20. Hur SE, Lee JY, Moon HS and Chung HW: Polymorphisms of the genes encoding the GSTM1, GSTT1 and GSTP1 in Korean women: No association with endometriosis. Mol Hum Reprod 11: $15-19,2005$.

21. Aban M, Ertunc D, Tok EC, Tamer L, Arslan M and Dilek S: Modulating interaction of glutathione-S-transferase polymorphisms with smoking in endometriosis. J Reprod Med 52 715-721, 2007.

22. Kim SH, Choi YM, Lee GH, Hong MA, Lee KS, Lee BS, Kim JG and Moon SY: Association between susceptibility to advanced stage endometriosis and the genetic polymorphisms of aryl hydrocarbon receptor repressor and glutathione-S-transferase T1 genes. Hum Reprod 22: 1866-1870, 2007.

23. Cao YH, Yao L, Wang D and Han P: Relationship between endometriosis and glutathione S-transferase N1, T1 and P1 genetic polymorphism. Maternal and Child Health Care of China 24 805-814, 2009 (In Chinese).

24. Wu HL, Feng D and Liu PX: Relationship between glutathione S-transferase M1 gene polymorphism and susceptibility to endometriosis. Yi Xue Lin Chuang Yan Jiu 26: 764-766, 2009 (In Chinese)

25. Huang PC, Tsai EM, Li WF, Liao PC, Chung MC, Wang YH and Wang SL: Association between phthalate exposure and glutathione S-transferase M1 polymorphism in adenomyosis, leiomyoma and endometriosis. Hum Reprod 25: 986-994, 2010.

26. Hosseinzadeh Z, Mashayekhi F and Sorouri ZZ: Association between GSTM1 gene polymorphism in Iranian patients with endometriosis. Gynecol Endocrinol 27: 185-189, 2011.

27. Wu CH, Guo CY, Yang JG, Tsai HD, Chang YJ, Tsai PC, Hsu CC and Kuo PL: Polymorphisms of dioxin receptor complex components and detoxification-related genes jointly confer susceptibility to advanced-stage endometriosis in the Taiwanese Han population. Am J Reprod Immunol 67: 160-168, 2012.

28. Seifati SM, Parivar K, Aflatoonian A, Dehghani Firouzabadi R and Sheikhha MH: No association of GSTM1 null polymorphism with endometriosis in women from central and southern Iran. Iran J Reprod Med 10: 23-28, 2012.

29. Matsuzaka Y, Kikuti YY, Goya K, Suzuki T, Cai LY, Oka A, Inoko H, Kulski JK, Izumi S and Kimura M: Lack of an association human dioxin detoxification gene polymorphisms with endometriosis in Japanese women: Results of a pilot study. Environ Health Prev Med 17: 512-517, 2012.

30. Chang Y: Association studies of the relationship between GSTM1, GSTT1 gene polymorphism and susceptibility to endometriosis in women of Han nationality in Hunan province. Masters thesis, Central South University, 2007. http://cdmd.cnki. com.cn/Article/CDMD-10533-2007172085.htm (In Chinese).

31. Sachan S, Nair RR, Khanna A and Singh K: CYP1A1 and GSTM1 genes polymorphism and its association with endometriosis: A pilot study. Asian Pacific Journal of Reproduction 2: 297-300, 2013

32. Baranova $\mathrm{H}$, Canis $M$, Ivaschenko $\mathrm{T}$, Albuisson $\mathrm{E}$, Bothorishvilli R, Baranov V, Malet P and Bruhat MA: Possible involvement of arylamine $\mathrm{N}$-acetyltransferase 2, glutathione S-transferases M1 and T1 genes in the development of endometriosis. Mol Hum Reprod 5: 636-641, 1999.
33. Baranov VS, Ivaschenko T, Bakay B, Aseev M, Belotserkovskaya R, Baranova H, Malet P, Perriot J, Mouraire P, Baskakov VN, et al: Proportion of the GSTM1 0/0 genotype in some Slavic populations and its correlation with cystic fibrosis and some multifactorial diseases. Hum Genet 97: 516-520, 1996.

34. Baranov VS, Ivashchenko TE, Shved NIu, Iarmolinskata MI, Sel'kov SA, Gorbushin SM, Savitskiı̌ GA, Malet P, Kanis M, Bruhat $\mathrm{M}$ and Baranova E: Genetic factors of predisposition to endometriosis and response to its treatment. Genetika 35: 243-248, 1999 (In Russian).

35. Hadfield RM, Manek S, Weeks DE, Mardon HJ, Barlow DH and Kennedy SH; OXEGENE Collaborative Group: Linkage and association studies of the relationship between endometriosis and genes encoding the detoxification enzymes GSTM1, GSTT1 and CYP1A1. Mol Hum Reprod 7: 1073-1078, 2001.

36. Baxter SW, Thomas EJ and Campbell IG: GSTM1 null polymorphism and susceptibility to endometriosis and ovarian cancer. Carcinogenesis 22: 63-65, 2001.

37. Ivashchenko TE, Shved NIu, Kramareva NA, Aŭlamazian EK and Baranov VS: Analysis of the polymorphic alleles of genes encoding phase 1 and phase 2 detoxication enzymes in patients with endometriosis. Genetika 39: 525-529, 2003 (In Russian).

38. Arvanitis DA, Koumantakis GE, Goumenou AG, Matalliotakis IM, Koumantakis EE and Spandidos DA: CYP1A1, CYP19, and GSTM1 polymorphisms increase the risk of endometriosis. Fertil Steril 79 (Suppl 1): 702-709, 2003.

39. Vichi S, Medda E, Ingelido AM, Ferro A, Resta S, Porpora MG, Abballe A, Nisticò L, De Felip E, Gemma S and Testai E: Glutathione transferase polymorphisms and risk of endometriosis associated with polychlorinated biphenyls exposure in Italian women: A gene-environment interaction. Fertil Steril 97: 1143-1151.e3, 2012.

40. Bischoff FZ, Marquez-Do D, Dang D, Carson SA, Buster JE and Simpson JL: NAT2 and GSTM1 DNA polymorphisms: Increased GSTM1 (active) genotypes in endometriosis. Fertil Steril 77 (Suppl 1): S17, 2002.

41. Trabert B, Schwartz SM, Peters U, De Roos AJ, Chen C, Scholes D and Holt VL: Genetic variation in the sex hormone metabolic pathway and endometriosis risk: An evaluation of candidate genes. Fertil Steril 96: 1401-1406.e3, 2011.

42. De Carvalho CV, D'Amora P, Schor E, Baracat EC, Girão MJC and Da Silva IDG: GSTM1 polymorphism analysis in patients with mild and severe endometriosis. Fertil Steril 82 (Suppl 2): S291, 2004

43. Frare AB, Barbosa AM, Costa IR, Souza SR, Silva RC, Bordin BM, Ribeiro Júnior CL and Moura KK: GSTM1 and GSTT1 polymorphisms in endometriosis in women from Goiás, Brazil. Genet Mol Res 12: 2764-2770, 2013.

44. Arvanitis DA, Goumenou AG, Matalliotakis IM, Koumantakis EE and Spandidos DA: Low-penetrance genes are associated with increased susceptibility to endometriosis. Fertil Steril 76: 1202-1206, 2001

45. Liu Y and Xu LZ: Meta-analysis of association between GSTM1 gene polymorphism and cervical cancer. Asian Pac J Trop Med 5: 480-484, 2012

46. Sergentanis TN and Economopoulos KP: GSTT1 and GSTP1 polymorphisms and breast cancer risk: A meta-analysis. Breast Cancer Res Treat 121: 195-202, 2010.

47. García-Closas M, Malats N, Silverman D, Dosemeci M, Kogevinas M, Hein DW, Tardón A, Serra C, Carrato A, García-Closas R, et al: NAT2 slow acetylation, GSTM1 null genotype, and risk of bladder cancer: results from the Spanish Bladder Cancer Study and meta-analyses. Lancet 366: 649-659, 2005.

48. Chen B, Cao L, Zhou Y, Yang P, Wan HW, Jia GQ, Liu L and Wu XT: Glutathione S-transferase T1 (GSTT1) gene polymorphism and gastric cancer susceptibility: A meta-analysis of epidemiologic studies. Dig Dis Sci 55: 1831-1838, 2010.

49. Wang H, Zhou Y, Zhuang W, Yin YQ, Liu GJ, Wu TX, Yao X, Du L, Wei ML and Wu XT: Glutathione S-transferase M1 null genotype associated with gastric cancer among Asians. Dig Dis Sci 55: 1824-1830, 2010

50. Das P, Shaik AP and Bammidi VK: Meta-analysis study of glutathione-S-transferases (GSTM1, GSTP1, and GSTT1) gene polymorphisms and risk of acute myeloid leukemia. Leuk Lymphoma 50: 1345-1351, 2009.

51. Nelson HH, Wiencke JK, Christiani DC, Cheng TJ, Zuo ZF, Schwartz BS, Lee BK, Spitz MR, Wang M, Xu X, et al: Ethnic differences in the prevalence of the homozygous deleted genotype of glutathione S-transferase theta. Carcinogenesis 16: 1243-1245, 1995. 